\title{
Erratum to: Recording of hormone therapy and breast density in breast screening programs: summary and recommendations of the International Cancer Screening Network
}

\author{
Brian Cox $\cdot$ Rachel Ballard-Barbash $\cdot$ Mireille Broeders • \\ Emily Dowling $\cdot$ Nea Malila $\cdot$ Rene Shumak . \\ Stephen Taplin · Diana Buist $\cdot$ Diana Miglioretti
}

Published online: 15 June 2010

(C) Springer Science+Business Media, LLC. 2010

\section{Erratum to: Breast Cancer Res Treat \\ DOI 10.1007/s10549-010-0893-0}

Authors regret to inform that, due to an unfortunate oversight, some data from the Swiss breast screening programme was omitted. The second and third sentences of the second paragraph of the Results should read.
Of the 29 countries approached, 8 programs recorded the use of prescribed HT at each screening episode (Table 1). Five of the eight also sought information about ever use of HT with four of the five, and one other, recording whether combined HT or progestin alone was prescribed.

The corrected Tables 1 and 2 are given as follows.

The online version of the original article can be found under doi:10.1007/s10549-010-0893-0.

\section{B. $\operatorname{Cox}(\square)$}

Hugh Adam Cancer Epidemiology Unit, Department of Preventive and Social Medicine, Dunedin School of Medicine, University of Otago, Dunedin 9054, New Zealand

e-mail: brian.cox@otago.ac.nz

R. Ballard-Barbash · E. Dowling · S. Taplin Applied Research Program, Division of Cancer Control and Population Sciences, National Cancer Institute, Bethesda, MD 20892, USA

\section{Broeders}

Department of Epidemiology, Biostatistics and Health

Technology Assessment, Radboud University Nijmegen Medical

Centre, Nijmegen 6500 HB, The Netherlands
N. Malila

Mass Screening Registry, Finnish Cancer Registry, Helsinki 00130, Finland

N. Malila

Tampere School of Public Health, University of Tampere,

Tampere, Finland

R. Shumak

Ontario Breast Screening Program, Cancer Care Ontario,

Toronto, ON MN 6G9, Canada

D. Buist · D. Miglioretti

Group Health Research Institute, Seattle, WA 98101, USA 
Table 1 Self-reported information on hormone therapy use in breast cancer screening programs: results from the International Cancer Screening Network

\begin{tabular}{|c|c|c|c|c|c|c|c|c|}
\hline Country & $\begin{array}{l}\text { At current screen } \\
\text { HT use recorded }\end{array}$ & $\begin{array}{l}\text { Current } \\
\text { use of } \\
\text { HRT }\end{array}$ & $\begin{array}{l}\text { Ever HT } \\
\text { use }\end{array}$ & $\begin{array}{l}\text { Combined HT and progestin } \\
\text { alone recorded separately }\end{array}$ & $\begin{array}{l}\text { Age } \\
\text { started }\end{array}$ & $\begin{array}{l}\text { Duration } \\
\text { in years }\end{array}$ & $\begin{array}{l}\text { Linkage } \\
\text { possible }\end{array}$ & $\begin{array}{l}\text { Menopausal state } \\
\text { recorded at screen }\end{array}$ \\
\hline Australia & Yes & Yes & & & & & Yes & \\
\hline Canada & Yes & Yes & Yes & Yes & No & No & Yes & Yes $^{\mathrm{a}}$ \\
\hline Denmark & Yes & Yes & & & & & Yes & \\
\hline Finland & Yes & Yes & Yes & Yes & & & Yes & \\
\hline Israel & Yes & Yes & Yes & & Yes & & & Yes \\
\hline $\begin{array}{l}\text { New } \\
\text { Zealand }\end{array}$ & No & No & No & No & No & No & Yes & No \\
\hline Norway & Yes & Yes & Yes & Yes & Yes & & Yes & \\
\hline Switzerland & Yes & Yes & Yes & Yes & Yes & Yes & No & Yes \\
\hline $\begin{array}{l}\text { United } \\
\text { States }^{\mathrm{b}}\end{array}$ & Yes & Yes & $\begin{array}{l}\text { Some } \\
\text { women }\end{array}$ & Yes ${ }^{b}$ & $\begin{array}{l}\text { Some } \\
\text { women }\end{array}$ & $\begin{array}{l}\text { Some } \\
\text { women }\end{array}$ & Yes $^{c}$ & Yes $^{d}$ \\
\hline
\end{tabular}

a Only at first screen

b The US does not have a national screening program. Data were derived from the Breast Cancer Screening Consortium and only some registries collect the type of HT

c One center only

d Varies by program site

Table 2 Recording of mammographic breast density (BD) in breast cancer screening programs: results from the International Cancer Screening Network

\begin{tabular}{|c|c|c|c|c|c|c|c|c|}
\hline Country & $\begin{array}{l}\text { BD } \\
\text { collected } \\
\text { at screen }\end{array}$ & $\begin{array}{l}\text { Only at } \\
\text { first } \\
\text { screen }\end{array}$ & Measure used & $\begin{array}{l}\text { Available } \\
\text { by age } \\
\text { group }\end{array}$ & $\begin{array}{l}\text { Moving to } \\
\text { full-field } \\
\text { digital (FFD) }\end{array}$ & $\begin{array}{l}\text { Has FFD } \\
\text { changed } \\
\text { way BD is } \\
\text { measured }\end{array}$ & $\begin{array}{l}\text { Computerized } \\
\text { radiography } \\
\text { (CR) used }\end{array}$ & $\begin{array}{l}\text { Has CR } \\
\text { changed way } \\
\text { BD measured }\end{array}$ \\
\hline Australia & No & & & & Some women & & Some women & \\
\hline Denmark & Yes & & Fatty or mixed & Yes & $\begin{array}{l}\text { All women } \\
\text { since } 2007\end{array}$ & No & Not used & \\
\hline Canada & Yes & No & & & Some women & No & Some women & No \\
\hline Finland & Yes $^{a}$ & No & & & Some women & & Some women & \\
\hline Germany & Yes & & & & Some women & & Some women & \\
\hline Israel & Yes & & 4 descriptive categories & & Some women & & Some women & \\
\hline Japan & No & & & & Some women & & Some women & \\
\hline New Zealand & No & & & & Some women & & Some women & \\
\hline Norway & No ${ }^{b}$ & & $<30 \%, 30-70 \%,>70 \%$ & Yes & Some women & & Not used & \\
\hline Switzerland & Yes & No & 4 descriptive categories & Yes & Some women & No & & \\
\hline UK & No & & & & Some women & & Some women & \\
\hline United States $^{c}$ & Yes $^{d}$ & No & $\begin{array}{l}\text { BI-RADS, MRS, Wolfe, } \\
\text { dichotomous, other unnamed } \\
4 \text { category systems, continuous }\end{array}$ & Yes & $\begin{array}{l}\text { Some women } \\
\text { since } 2001\end{array}$ & No & Some women & \\
\hline Czech Republic & Yes & No & Tabar classification & Yes & Some women & & Some women & \\
\hline
\end{tabular}

\footnotetext{
a Historical data only and used Wolfe's classification

b Only recorded for women who were recalled

c The US does not have a national screening program. Data were derived from the Breast Cancer Screening Consortium

d BI-RADS is the majority, Wolfe no longer used 2000 forward, dichotomous no longer used 1999 forward
} 